\title{
Diagnosis and Therapy of Microscopic Colitis with Presence of Foamy Macrophages in Children
}

\author{
Jan Józefczuk ${ }^{1}$ and Bogdan Marian Woźniewicz ${ }^{2}$ \\ ${ }^{1}$ Pediatric Ward, Hospital in Sandomierz, Schinzla 13, 27-600 Sandomierz, Poland \\ ${ }^{2}$ Department of Pathology, The Children's Memorial Health Institute, Aleja Dzieci Polskich 20, 04-730 Warsaw, Poland
}

Correspondence should be addressed to Bogdan Marian Woźniewicz, b.wozniewicz@czd.waw.pl

Received 12 April 2011; Accepted 4 May 2011

Academic Editor: C.-Y. Chen

Copyright ( 2011 J. Józefczuk and B. M. Woźniewicz. This is an open access article distributed under the Creative Commons Attribution License, which permits unrestricted use, distribution, and reproduction in any medium, provided the original work is properly cited.

\begin{abstract}
We discuss the diagnosis of and efficacy 5-amino-2-hydroxybenzoic acid (5-ASA), Saccharomyces boulardii, or magnesium in therapy of microscopic colitis with presence of foamy macrophages. A basis for diagnosis and inclusion to the analysed group was presence of characteristic foamy macrophages in histopathological examination of hematoxylin and eosin-stained specimens collected from the large intestine, reviewed under $\times 200$ or $\times 320$ magnification. No statistically significant improvement was found following the use of 5-amino-2-dihydroxybenzoic acid in therapy of the disease. The use of Saccharomyces boulardii was associated with statistically significant improvement in clinical, endoscopic, and histopathological condition. Use of magnesium caused a histological, statistically significant improvement but failed to have any effect on the clinical and endoscopic presentation. In the group of children in whom no therapeutic intervention was provided, a statistically significant spontaneous clinical improvement was observed, but no statistically significant changes in endoscopic and microscopic condition were found.
\end{abstract}

\section{Introduction}

Typical inflammatory bowel disease (IBD), such as ulcerative colitis or Crohn's disease, is only a part of cases of persistent enteritis. Nonspecific IBDs are most often superficial processes, nondamaging to the structure of intestinal mucosa and submucosa. In the medical literature, they are usually referred to as microscopic enteritis [1-4].

Foamy colitis with presence of foamy macrophages within the intestinal lamina propria belongs to a group of microscopic inflammations in children. That recently separated disease requires determination of effective therapeutic methods $[5,6]$.

Observation of that type of inflammation, indicating a rather mild clinical course of the disease and no cases of its transformation into a typical inflammatory bowel disease (IBD) justified undertaking a prospective observation of children treated with 5-amino-2-hydroxybenzoic acid, with a probiotic (Saccharomyces boulardii), or with magnesium, and of a group of children who were not treated pharmacologically.
Evaluation of clinical, endoscopic, and microscopic effects of therapy with 5-amino-2-dihydroxybenzoic acid, Saccharomyces boulardii, or magnesium of children with microscopic colitis with presence of foamy macrophages and evaluation of a group of children with no therapeutic intervention.

\section{Material and Methods}

A basis for diagnosis of the disease was presence of macrophages that stained with hematoxylin and eosin formed a characteristic image of foamy (clear cell) macrophages, in the lamina propria of the large intestine.

Additional examinations using electron microscopy Jeol $100 \mathrm{CX}$ were performed in several cases. Sections of mucosa were fixed in cacodylate buffered $4 \%$ glutaraldehyde and then in osmium tetroxide $\left(\mathrm{OsO}_{4}\right)$ and analysed acc. to a typical procedure for Epon. Resulting ultrathin sections were additionally stained with lead citrate and uranyl acetate.

Uneven distribution and quantity of foamy macrophages constituted a basis for development of our own classification 
of microscopic changes, defining three levels of intensity:

(i) single cells on various levels of the lamina propria,

(ii) presence of aggregates composed of 3-10 cells,

(iii) foamy cells covering evenly the whole lamina propria.

That type of cells was absent in other layers of the large intestine, and their presence was not found in other sections of the alimentary tract.

In the process of identification of foamy macrophages, except for hematoxylin and eosin staining of serial specimens, also a PAS reaction was used, with and without diastase digestion.

Additionally, immunocytochemical tests using monoclonal sera from DAKO: anti-CD3(T cell), CD4(T cell), CD22 (B cell), CD31 (Endothelia), CD34 (precursor cell), CD 68 (Macrophages), allowing identification of cells occurring in an inflammatory process, were completed on specimens placed on hyalinized slides. Tests were also completed to detect the PCNA-proliferation antigen, Ki67-mitotic activity, chromogranin, synaptophysin, neurofilament, actin, P53 mutations, GFAP glial marker, TNF alpha, IL2, and mucicarmin.

Tests were competed to detect intestinal pathogens (Salmonella, Shigella, Camphylobacter, Escherichia coli, Yersnia, Clostridium difficile) and Giardia infection.

Tests for enteroviruses were performed on cell lines (Hep 2 and RD) in the Department of Virusology of the National Health Institute, using WHO-recommended methods. Presence of rotaviruses was tested with the latex reaction, using the Slidex Rota-Kit, and completing serological tests with ELISA, using Rabbit Anti-Rotavirus Human reagents from DAKO.

Children with presence of foamy macrophages confirmed in histopathological specimens and who had a clinical and endoscopic changes characteristic for colitis were randomized to individual therapeutic groups of a prospective, open trial realised from 1999 to 2009.

In the analysis, there were included 144 children aged from 3 to 18 years. For 12 months, children received 5-amino-2-hydroxybenzoic acid (5-ASA) at dose of 20$25 \mathrm{mg} / \mathrm{kg}$ b.w. $/ 24 \mathrm{~h}$ (38 children-group A), Saccharomyces boulardii at dose of $15-20 \mathrm{mg} / \mathrm{kg}$ b.w. $/ 24 \mathrm{~h}$ (35 children-group B), or magnesium $5-10 \mathrm{mg} / \mathrm{kg}$ b.w. $/ 24 \mathrm{~h}$ (35 children-group D).

Children who received no treatment constituted a control group (36 children-group C).

Clinical and endoscopic examinations were performed at the Pediatric Ward, and microscopic and immunocytochemical examinations-in the Department of Pathology in the Children's Memorial Health Institute in Warsaw.

Clinical evaluation included general feeling, presence of abdominal pain and its intensity, number of bowel movements per week, presence of blood in stool, fever, presence of extraenteral symptoms, ESR, haemoglobin concentration, and nutritional status expressed as Cole and Stanfield index.

Clinical activity index (CAI) acc. to Rachmilewitz was used for evaluation of clinical presentation of analysed children [7].
The level of endoscopic lesions was determined by evaluation of vascular pattern, granulation, and mucosa proneness for bleeding, as well as presence of mucus, pus, erosions, and ulcerations on the surface of colic mucosa. The clinical activity index (CAI) acc. to Rachmilewitz was then applied [7].

Evaluation of clinical and endoscopic changes was performed at diagnosis and following 12 months of therapy and observation of each analysed child.

Due to nonparametric distribution of sets, a sign test was used for statistical evaluation of results (Statistica software).

\section{Results and Discussion}

At diagnosis, histopathological examination of bioptates collected from the large intestine revealed presence of large, clear, foamy cells in the lamina propria of the mucosa. Those cells appeared single or in agglomerates, covering the whole width of the lamina propria-from the covering epithelium to the base of intestinal crypts and the level of muscularis mucosa. (Figure 1)

Immunocytochemical tests indicated a strong positive reaction with the CD68 antibody, typical for activated macrophages. Foamy macrophages showed a positive PAS reaction with periodic acid and Shiff's reagent. However, the reaction was negative following digestion with diastase. Immunocytochemical reaction to neurofilament was negative. Using the marker PCNA-proliferation antigen an increased activity was demonstrated only in cryptic epithelium. No increased activity was found in epithelium surrounding crypts (Figure 2).

Other numerous, mentioned in the Methods section, immunocytochemical markers gave a negative result in tests with foamy macrophages (Table 1).

Before statistical analysis of results of clinical, endoscopic, and microscopic evaluation results for three study groups, they were evaluated at the time of intervention. Hypothesis of equality of those three groups (A, B, C, D) in clinical, endoscopic, and microscopic evaluation cannot be dismissed (Whitney-Mann test) (Figure 3).

In the group $\mathrm{A}$, for 12 months, children received Masalazine (5 amino-2-hydroxybenzoic acid). Clinical, endoscopic, and microscopic evaluation of the group A, treated with 5-amino-2-hydroxybenzoic acid, showed no significant improvement, neither in clinical, endoscopic, nor in microscopic presentation (Figure 4).

In the group B, Saccharomyces boulardii was used for 12 months. In the group B of children treated with Saccharomyces boulardii the 12-month period of therapy caused a clinical, endoscopic, and microscopic, statistically significant improvement (Figure 5).

In the group $\mathrm{C}$ of children with microscopic colitis with presence of foamy macrophages, no therapy was applied.

In the group $\mathrm{C}$ of untreated children the clinical evaluation showed statistically significant improvement, and the endoscopic and microscopic evaluation showed no regression of pathological lesions (Figure 6).

Children in the group D had magnesium administered for 12 months. Statistical analysis of 12-month magnesium 


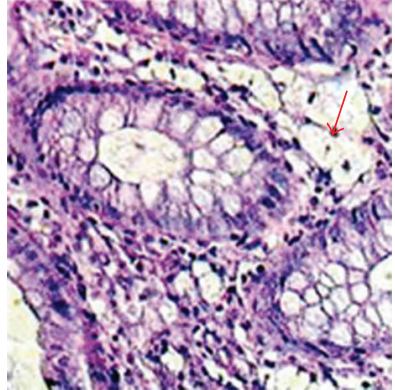

(a)

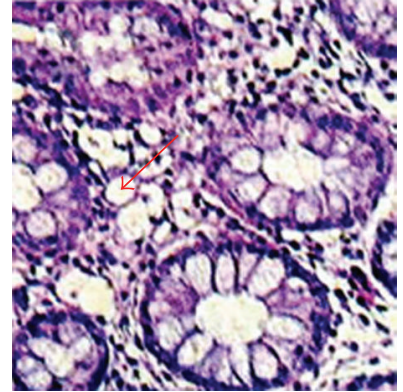

(b)

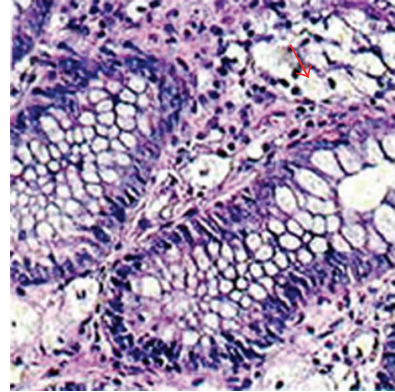

(c)

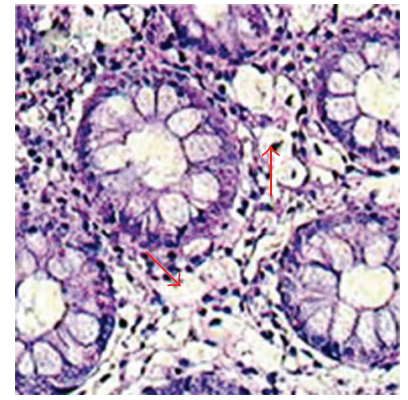

(d)

Figure 1: (a-d) Microscopic features of colitis with presence of foamy macrophages—characteristic large, clear cells with a small nucleus, single or aggregated within the lamina propria. HE staining. Magnification $\times 200$.

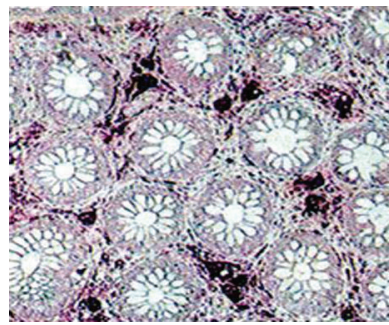

(a)

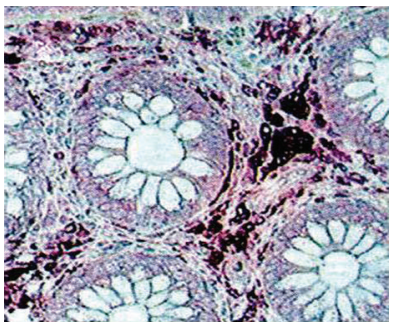

(b)

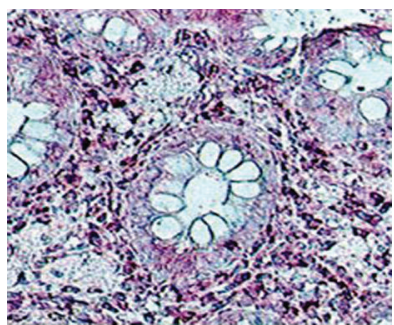

(c)

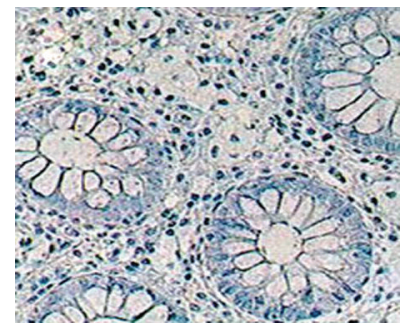

(d)

FIgURE 2: (a-d) Immunochemistry. (a-b) positive with a serum marking macrophages. C-negative to limfocytes T. D-neurofilament's negative reaction. Magnification $\times 200(a) \times 320(b, c, d)$.

TABLE 1: Expression of immunocytochemical markers in the microscopic colitis with presence of foamy macrophages.

\begin{tabular}{ll}
\hline Macrophages foamy & Marker \\
\hline Negative & $\mathrm{CD}_{3}($ Cell T) \\
Negative & $\mathrm{CD}_{4}($ Cell T) \\
Negative & $\mathrm{CD}_{22}$ (Cell B) \\
Negative & $\mathrm{CD}_{34}$ (Cell precursor) \\
Negative & $\mathrm{CD}_{31}$ (Entothelial) \\
Positive & $\mathrm{CD}_{68}$ (Macrophages) \\
Negative & $\mathrm{Ki}_{67}$ mitotic activity \\
Negative & $\mathrm{Chromogranin}$ \\
Negative & Synaptophysin \\
Negative & Neurofilament \\
Negative & Actin \\
Negative & $\mathrm{P}_{53}$ mutations \\
Negative & $\mathrm{GFAP}$ glial marker \\
Negative & $\mathrm{TNF}$ alpha \\
Positive & $\mathrm{PAS}$ \\
Positive & Osmium tetraoxide \\
Negative & $\mathrm{IL} 2$ \\
Negative & Mucicarmin \\
\hline
\end{tabular}

therapy shows microscopic and clinical improvement, but without any endoscopic improvement. Microscopic diagnosis of that unspecific inflammatory disease is relatively easy (Figure 7).
Presence of characteristic foamy macrophages observed at $\times 200$ and $\times 320$ magnification in hematoxylin and eosinstained specimens is a crucial diagnostic factor $[5,6]$. The disease is not associated with destruction of glands or their restructure. The lamina propria is not destroyed by lymphocytic infiltration, characteristic for inflammations belonging to the group of IBD. Presence of foamy macrophages in examined histopathological specimens excludes diagnosis of Colitis ulcerosa or Crohn's disease.

In IBD-type inflammations, proliferation of the mononuclear-phagocytic line is characterised by a positive expression of TNF-alpha and IL-beta, absent in our examinations. Microscopic evaluation of properly collected and prepared specimens, but observed under a routinely used low magnification, is a reason for underdiagnosis of the disease in other centres. Observation of the whole group of children confirms previous findings on mild and moderate course of that type of inflammation $[4,6]$.

Children left without any therapeutic intervention show no endoscopic or microscopic progression of lesions, and an observed surprising clinical improvement indicates an ability for independent regeneration of the intestine in that disease.

Existing reports indicate efficacy of therapy of mild colitis with probiotics. Successful attempts were made in therapy of ulcerative colitis and even of Crohn's disease [8-12]. Part of favourable therapeutic effects of monotherapy with probiotics could be a result of erroneous qualification of microscopic colitis to a group of mild IBD. It seems that probiotics may be useful as supplementary therapy, but their 

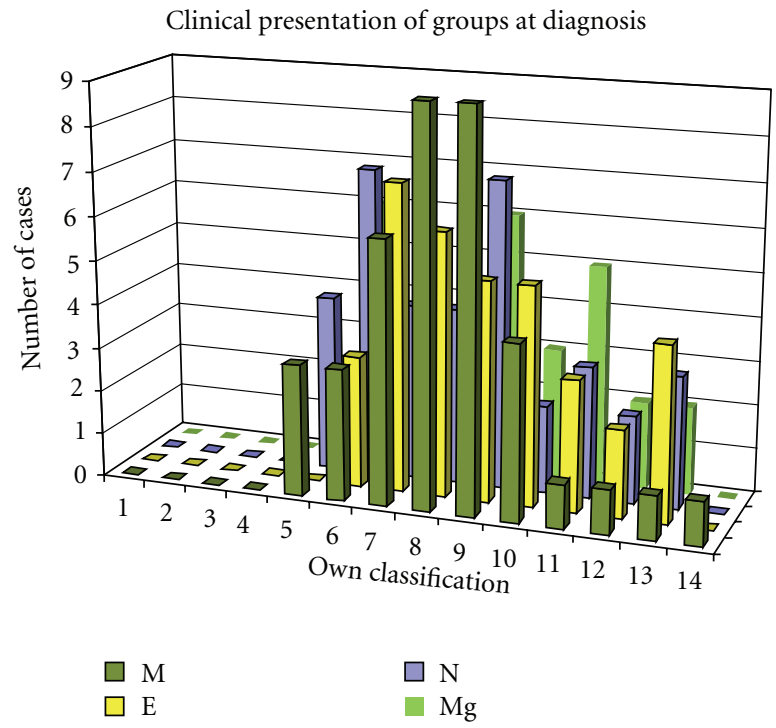

Statistica U Mann-Whitney test: hypothesis of equality of distribution cannot be dismissed

(a)

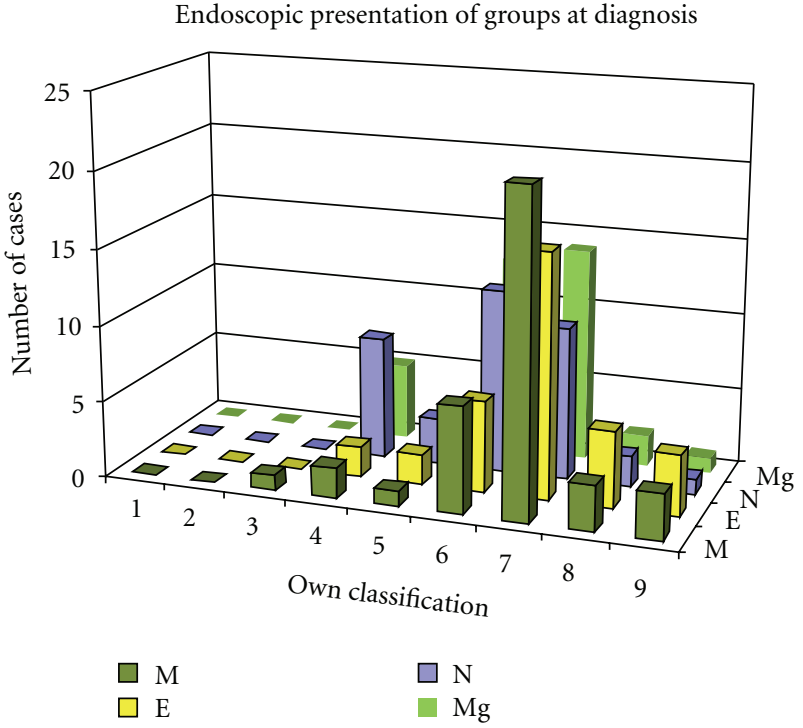

Statistica U Mann-Whitney test: hypothesis of equality of distribution cannot be dismissed

(b)

Microscopic presentation of groups at diagnosis

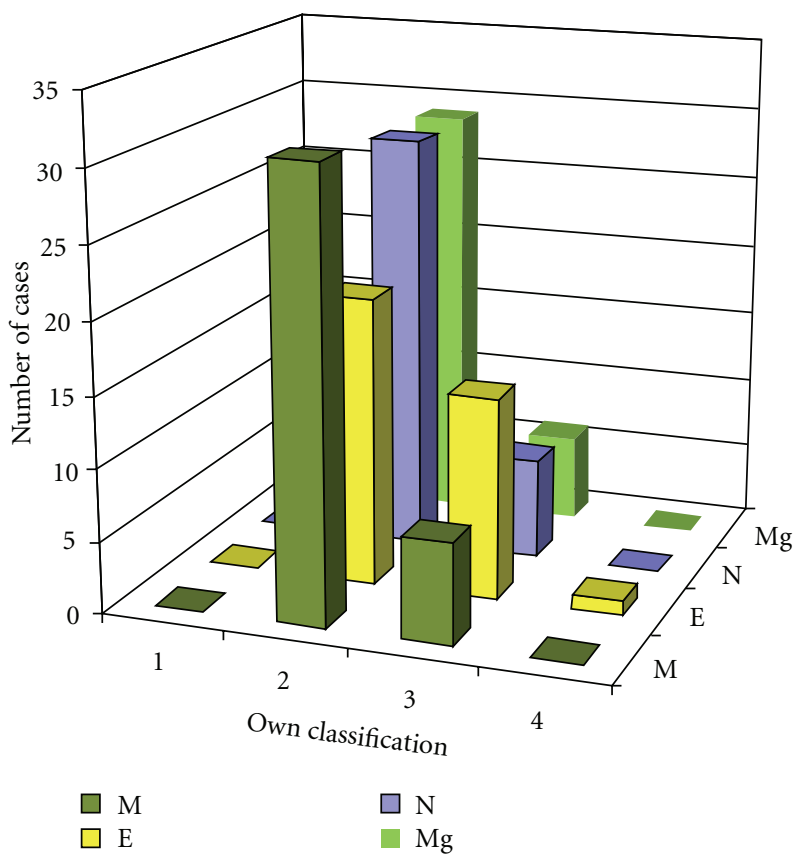

Statistica U Mann-Whitney test:

hypothesis of equality of distribution cannot be dismissed

(c)

Figure 3: Clinical, endoscopic, and microscopic evaluation at diagnosis (Whitney-Mann test).

use as a monotherapy is at least risky, particularly in the Crohn's disease.

A favourable clinical effect of Saccharomyces boulardii demonstrated in this trial, and confirmed in control endoscopic and microscopic examinations, may be a result of a trophic effect of carbohydrates, B group vitamins $\left(B_{1}, B_{2}, B_{6}\right.$,
$\left.B_{12}\right)$, folic acid, and pantoteic acid contained in that probiotic on enterocytes [8].

Nicotinamide included in Saccharomyces boulardii may reduce free oxygen radicals causing intensification of the inflammatory process, and numerous enzymes contained there; for example, proteases and disaccharidases may cause 


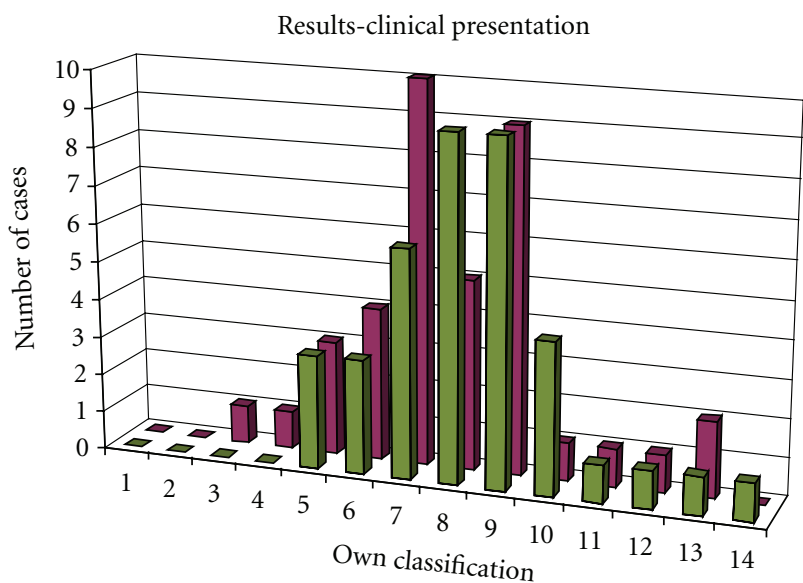

Before treatment

After treatment

Statistica ranked sign test: $(Z=2.085144, P=0.037056)$ hypothesis of equality of distributions cannot be dismissed

(a)

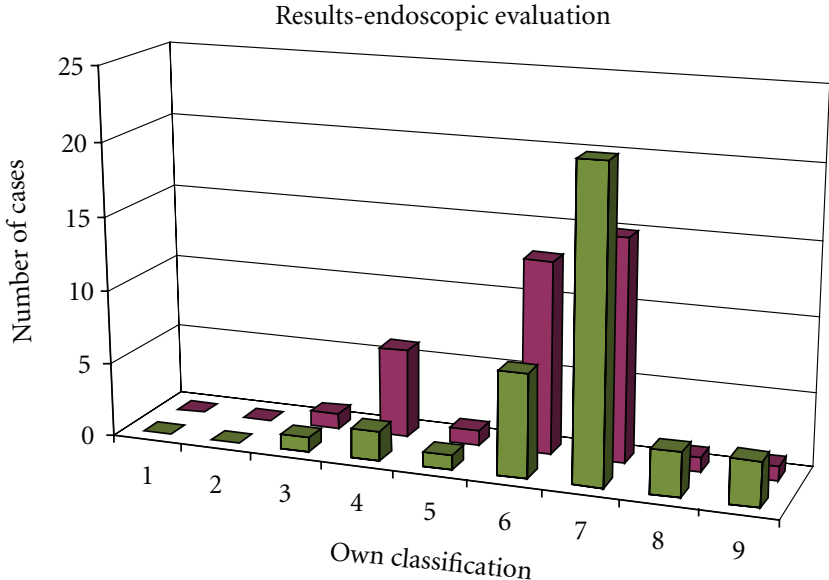

Before treatment

After treatment

Statistica ranked sign test: $(Z=1.835326, P=0.066457)$ hypothesis of equality of distributions cannot be dismissed

(b)

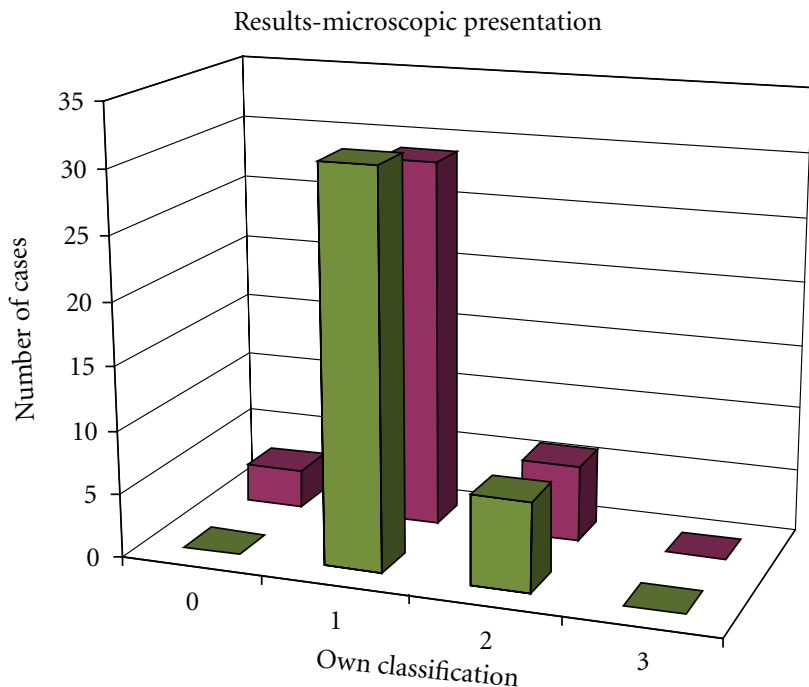

Before treatment

After treatment

Statistica ranked sign test: $(Z=0.948683, P=0.342782)$ hypothesis of equality of distributions cannot be dismissed

(c)

FIGURE 4: Clinical, endoscopic, and microscopic evaluation of children treated with 5-amino-2-hydroxybenzoic acid (group A).

digestion of protein content of foamy macrophages and be responsible for reduction of their count, demonstrated in control microscopic examinations $[8,13]$.

A favourable therapeutic effect of Saccharomyces boulardii may be also a result of its confirmed immunostimulatory and anti-inflammatory effect, significant for limitation and reduction of the inflammatory process in the intestine [13-15].
No favourable therapeutic effect of 5-amino-2-dihydroxybenzoic acid and relatively mild course of the disease justify resignation from therapy with Mesalazine (5-amino-2dihydroxybenzoic acid) in case of that type of colitis.

A favourable therapeutic effect of magnesium, manifested by regression of microscopic lesions, is most probably a result of supplementation of magnesium deficiency, resulting from erosion of soil and low content of the element in 


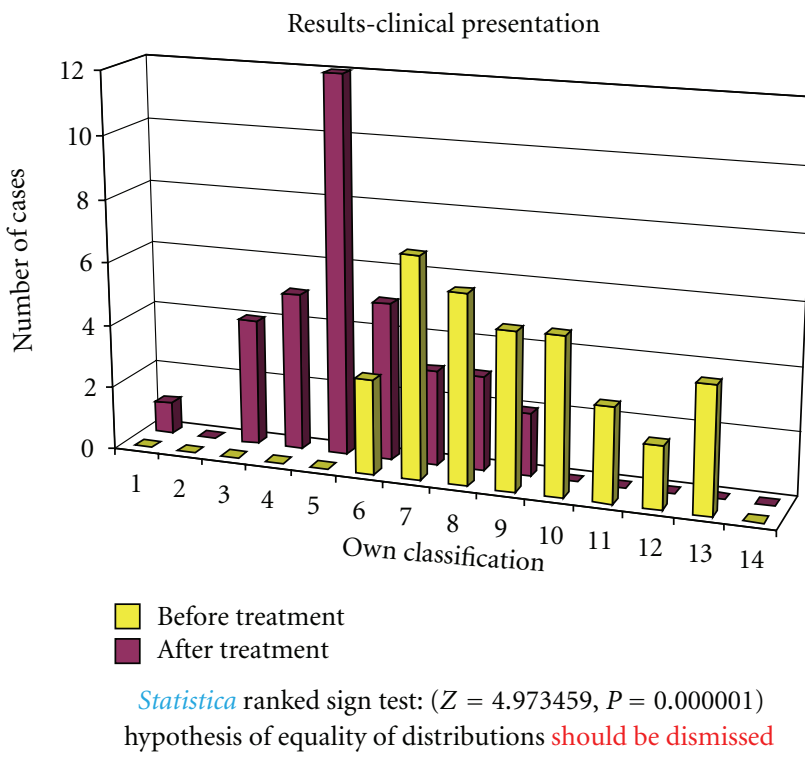

(a)

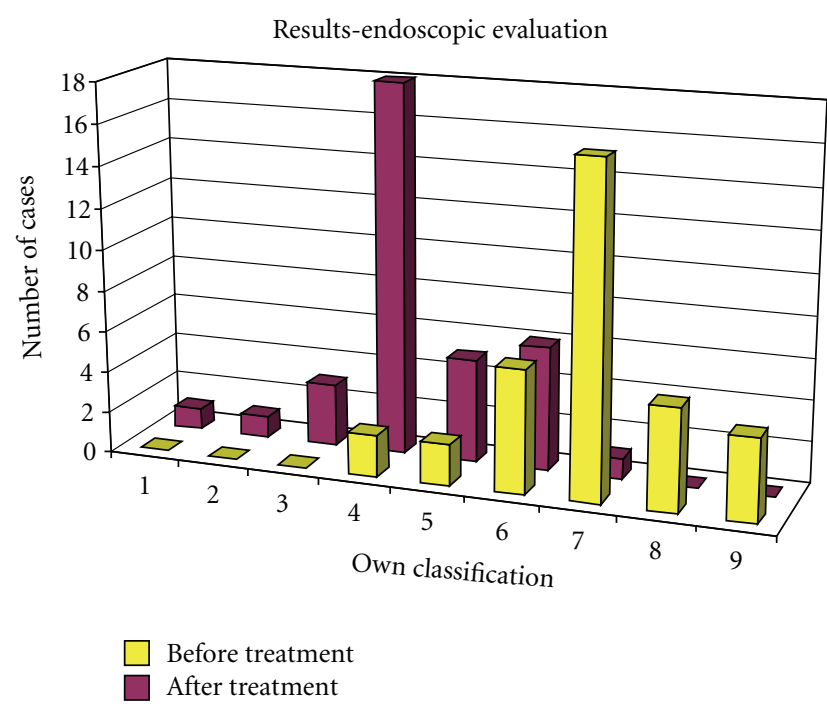

Statistica ranked sign test: $(Z=4.929503, P=0.000001)$ hypothesis of equality of distributions should be dismissed

(b)

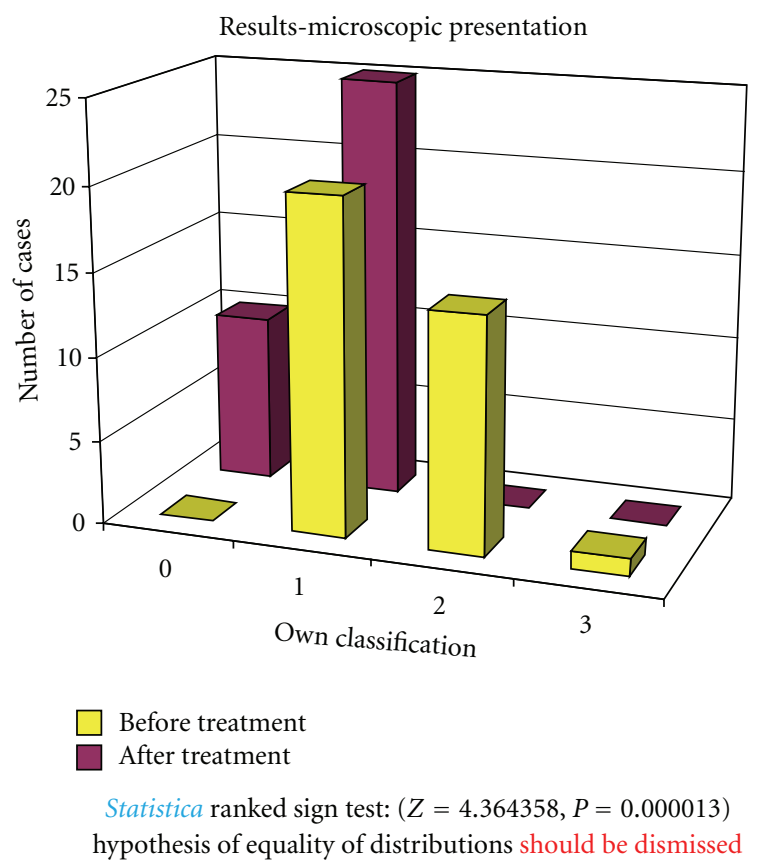

(c)

FIGURE 5: Clinical, endoscopic, and microscopic evaluation of children treated with Saccharomyces boulardii (group B).

food, and generally low level of supplementation. Besides, mucosa defects found in colitis intensify magnesium deficiency through increased apoptosis.

A favourable effect of magnesium treatment should be probably associated with activating effect of the element on enzymes necessary for synthesis and utilisation of highenergy compounds inside enterocytes. Further observation seems important, expecting development of endoscopic improvement. If it occurs, the microscopic improvement should be treated as a herald of the endoscopic improvement.

Systematic general paediatric and gastroenterological control, along with monitoring of number of foamy macrophages in microscopic examination, plays a very important role in diagnosis and therapy of that type of colitis [2]. The suggested own scale for evaluation of microscopic changes based on quantity of foamy macrophages in the lamina propia shows a complete correlation with the clinical and 


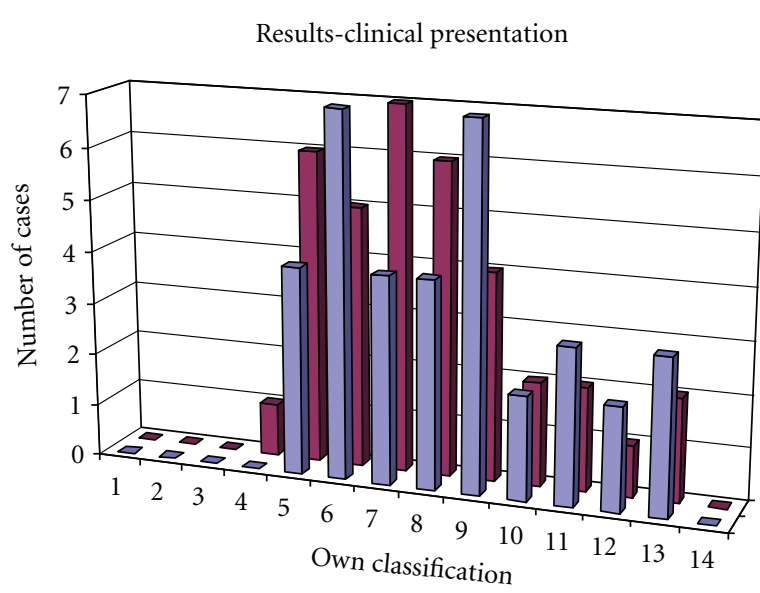

Before treatment

After treatment

Statistica ranked sign test: $(Z=2.752989, P=0.005905)$ hypothesis of equality of distributions should be dismissed

(a)
Results-endoscopic evaluation

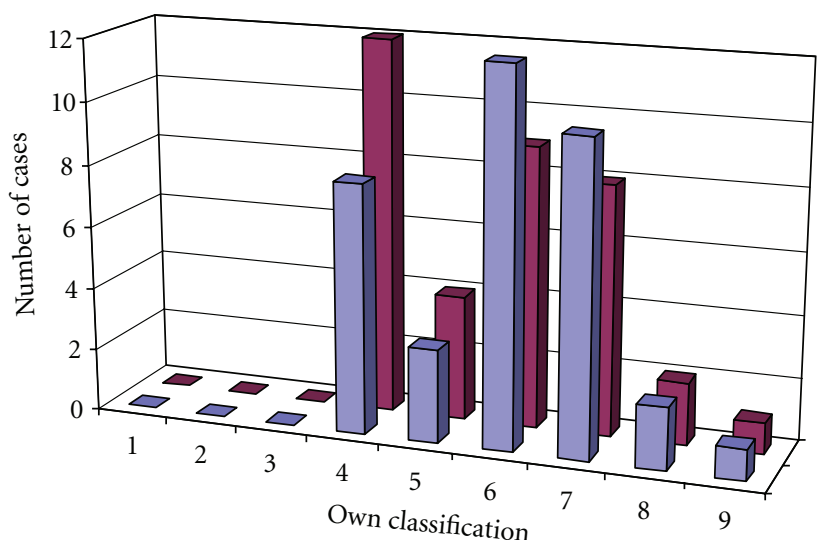

Before treatment

After treatment

Statistica ranked sign test: $(Z=1.109400, P=0.267258)$ hypothesis of equality of distributions should be dismissed

(b)

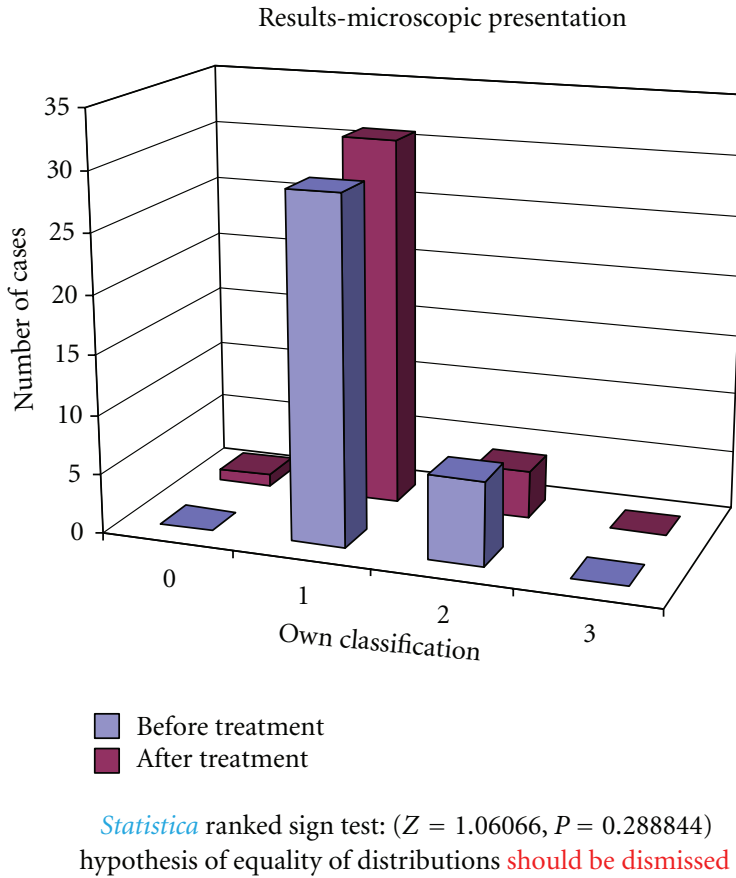

(c)

FIgURE 6: Clinical, endoscopic, and microscopic evaluation of untreated children (group C).

endoscopic evaluation, when analysing the therapeutic effect of Saccharomyces boulardii and 5-amino-2-dihydroxybenzoic acid and a correlation with the endoscopic presentation in case of no therapeutic intervention.

Prospective clinical observation, periodical endoscopic examinations of patients with that disease, will allow verification of currently available knowledge, and possibly will open new diagnostic and therapeutic horizons. Difficulties associated with unanimous classification of a significant part of colitis to a strictly defined group are a serious medical problem $[5,16]$. Classification of chronic colitis inadequate to actual condition may negatively influence therapy and prognosis in this large, socially important group of civilisation diseases. 
Results-clinical presentation

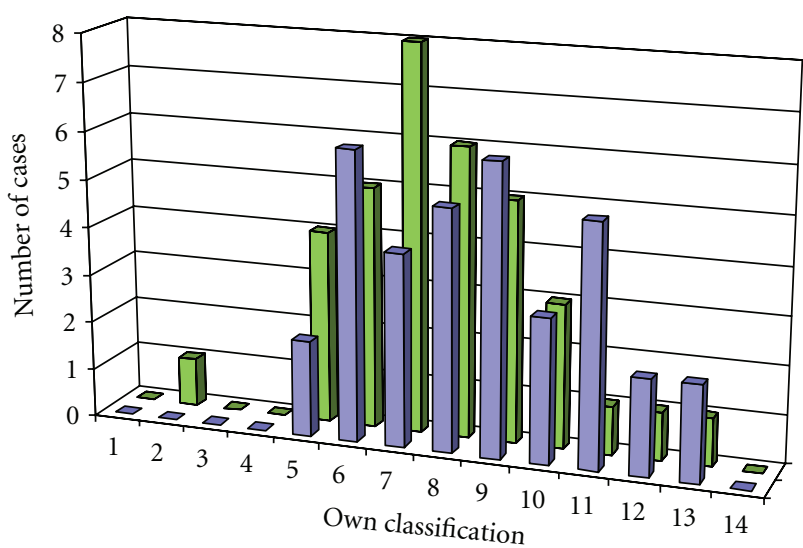

Before treatment

After treatment

Statistica sign test: $(Z=3.198011, P=0.001384)$ hypothesis of equality of distributions should be dismissed

(a)
Results-endoscopic evaluation

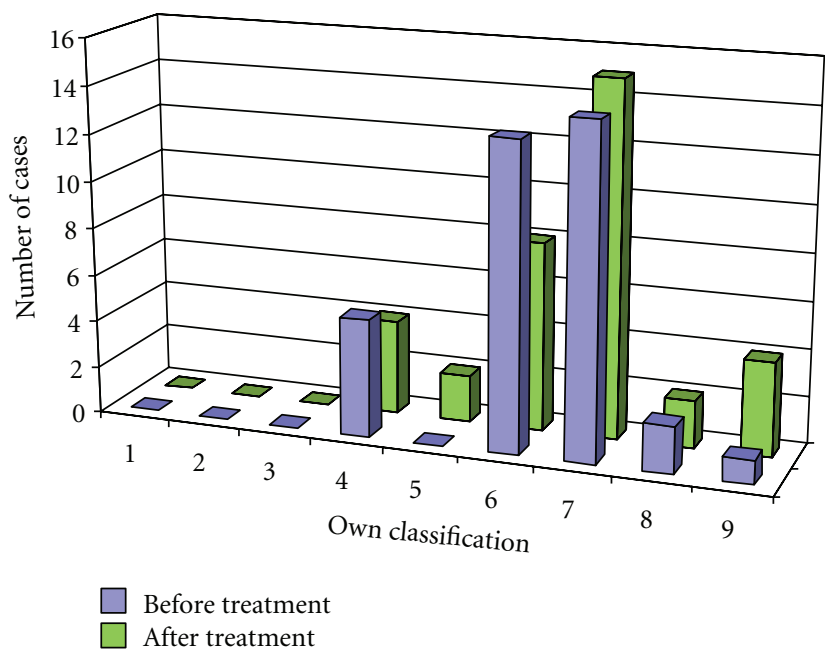

Statistica sign test: $(Z=2.25, P=0.024449)$ hypothesis of equality of distribution cannot be dismissed

(b)

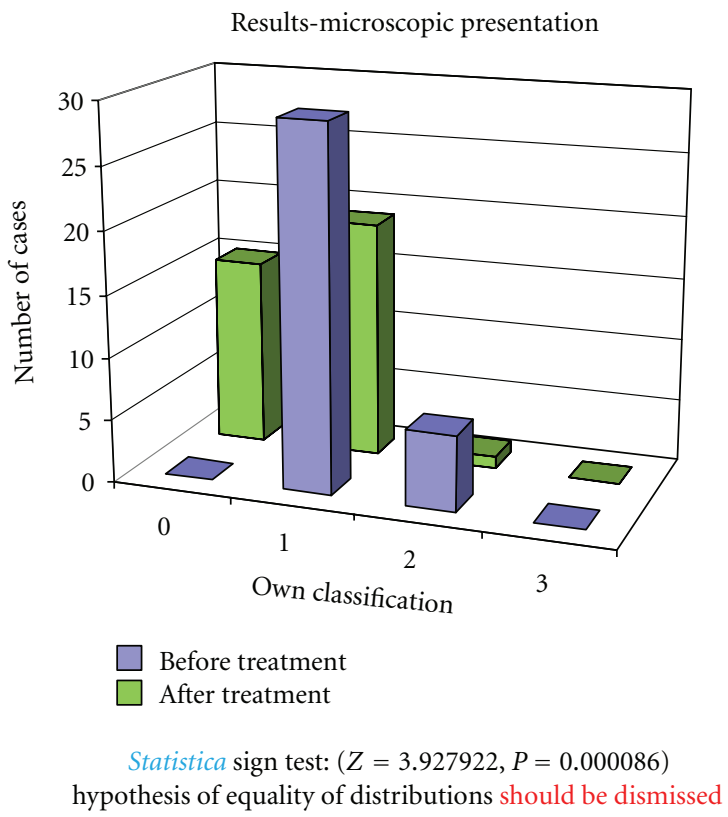

(c)

Figure 7: Clinical, endoscopic, and microscopic evaluation of children treated with magnesium (group D).

\section{Conclusions}

(1) Presence of foamy macrophages within the lamina propria of the large intestine is a deciding factor in diagnosis of that form of microscopic colitis.

(2) Identification of foamy macrophages is possible with routine hematoxylin and eosin staining and microscopic evaluation of histological specimens at $\times 200$ or $\times 320$ magnification.
(3) Efficacy of Saccharomyces boulardii in therapy of microscopic colitis with presence of foamy macrophages was demonstrated.

(4) No favourable therapeutic effect was achieved following use of 5-amino-2-dihydroxybenzoic acid (ASA-5) in case of the disease.

(5) The disease shows a tendency for spontaneous remission, but only in terms of clinical presentation. 
(6) Microscopic and clinical improvement found in case of magnesium therapy may be a herald of expected endoscopic improvement.

(7) Further paediatric and gastroenterological followup of children with that type of microscopic colitis is necessary.

\section{References}

[1] L. Librecht, R. Croes, and N. Ectors, "Microscopic colitis with giant cells," Histopatology, vol. 48, pp. 116-132, 2006.

[2] R. K. Yantiss and R. D. Odze, "Diagnostic difficulties in inflammatory bowel disease pathology," Histopathology, vol. 48, no. 2, pp. 116-132, 2006.

[3] B. F. Warren, C. M. Edwards, and S. P. L. Travis, "Microscopic colitis, classification and terminology," Histopathology, vol. 40, no. 4, pp. 374-376, 2002.

[4] J. Józefczuk, B. Woźniewicz, and W. Romańczuk, "Patologia i klinika nowej formy zapalenia jelita grubego u dzieci," Pediatria Współczesna. Gastroenterologia, Hepatologia i Żywienie Dziecka, vol. 5, no. 3, pp. 151-156, 2003.

[5] J. Józefczuk, B. Woźniewicz, and W. Romańczuk, "Clinicopathology of Foamy Cell Colitis (FCC), the new form noninflamatory bowel disease," Annals of Diagnostic Paediatric Pathology, vol. 5, pp. 71-74, 2001.

[6] J. Józefczuk and B. M. Wozniewicz, "Clear cell colitis: a form of microscopic colitis in children," World Journal of Gastroenterology, vol. 14, no. 2, pp. 231-235, 2008.

[7] J. Ryżko, J. Socha, and M. Woynarowski, "Validation of disease activity indexes Indexes for inflammatory disease," Surg. Child. Int., vol. 4, pp. 17-21, 1966.

[8] J. P. Buts and P. Bernasconi, "Saccharomyces boulardii: basic science and clinical applications in gastroenterology," Gastroenterology Clinics of North America, vol. 34, no. 3, pp. 515-532, 2005.

[9] O. Erdeve, U. Tiras, and Y. Dallar, "The probiotic effect of Saccharomyce boulardii in a pediatric age group," Journal of Tropical Pediatrics, vol. 50, no. 4, pp. 234-236, 2004.

[10] M. Guslandi, P. Giollo, and P. A. Testoni, "A pilot trial of Saccharomyces boulardii in ulcerative colitis," European Journal of Gastroenterology and Hepatology, vol. 15, no. 6, pp. 697-698, 2003.

[11] M. Guslandi, G. Mezzi, M. Sorghi, and P. A. Testoni, "Saccharomyces boulardii in maintenance treatment of Crohn's disease," Digestive Diseases and Sciences, vol. 45, no. 7, pp. 1462-1464, 2000.

[12] J. Ryżko, "Zastosowanie probiotyków i prebiotyków w leczeniu nieswoistych zapaleń jelit oraz zaburzeń czynnościowych jelita grubego," Pediatria Współczesna Gastroenterologia. Hepatologia i Żywienie Dziecka, vol. 4, no. 1, pp. 55-60, 2002.

[13] J. P. Buts, P. Bernasconi, J. P. Vaerman, and C. Dive, "Stimulation of secretory IgA and secretory component of immunoglobulins in small intestine of rats treated with Saccharomyces boulardii," Digestive Diseases and Sciences, vol. 35, no. 2, pp. 251-256, 1990.

[14] K. Plein and J. Hotz, "Therapeutic effects of Saccharomyces boulardii on mild residual symptoms in a stable phase of Crohn's disease with special respect to chronic diarrhea-a pilot study," Zeitschrift fur Gastroenterologie, vol. 31, no. 2, pp. 129-134, 1993.
[15] I. R. Sanderson, S. Boyle, C. B. Williams, and J. A. WalkerSmith, "Histological abnormalities in biopsies from macroscopically normal colonoscopies," Archives of Disease in Childhood, vol. 61, no. 3, pp. 274-277, 1986.

[16] K. Karolewska-Bochenek, I. Lazowska-Przeorek, P. Albrecht et al., "Epidemiology of inflammatory bowel disease among children in Poland," Digestion, vol. 79, no. 2, pp. 121-129, 2009. 


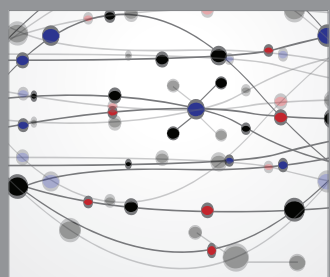

The Scientific World Journal
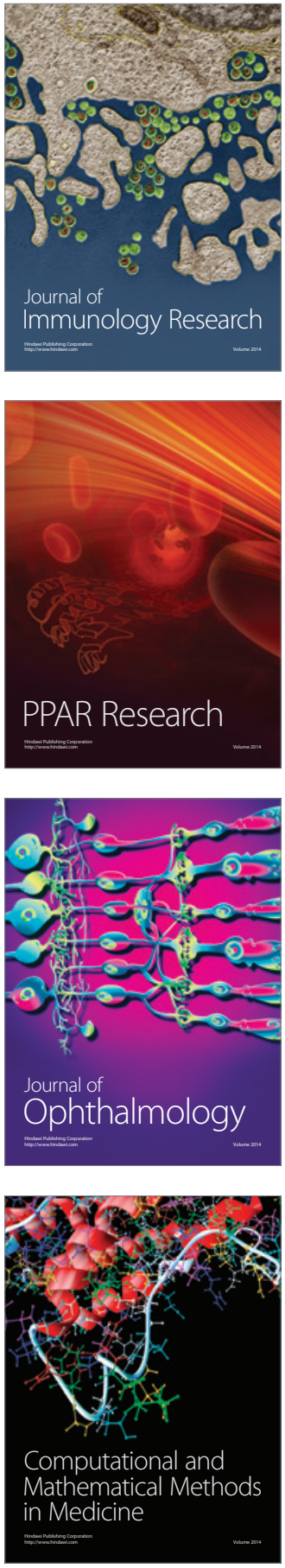

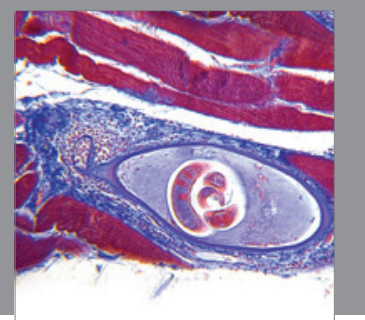

Gastroenterology

Research and Practice
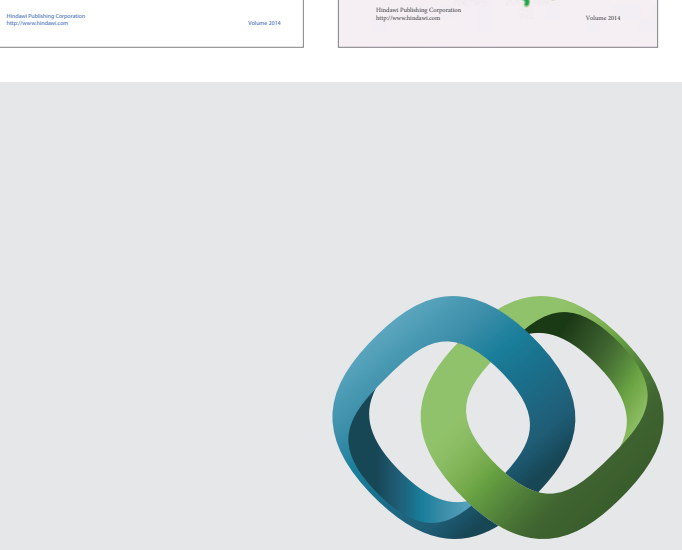

\section{Hindawi}

Submit your manuscripts at

http://www.hindawi.com
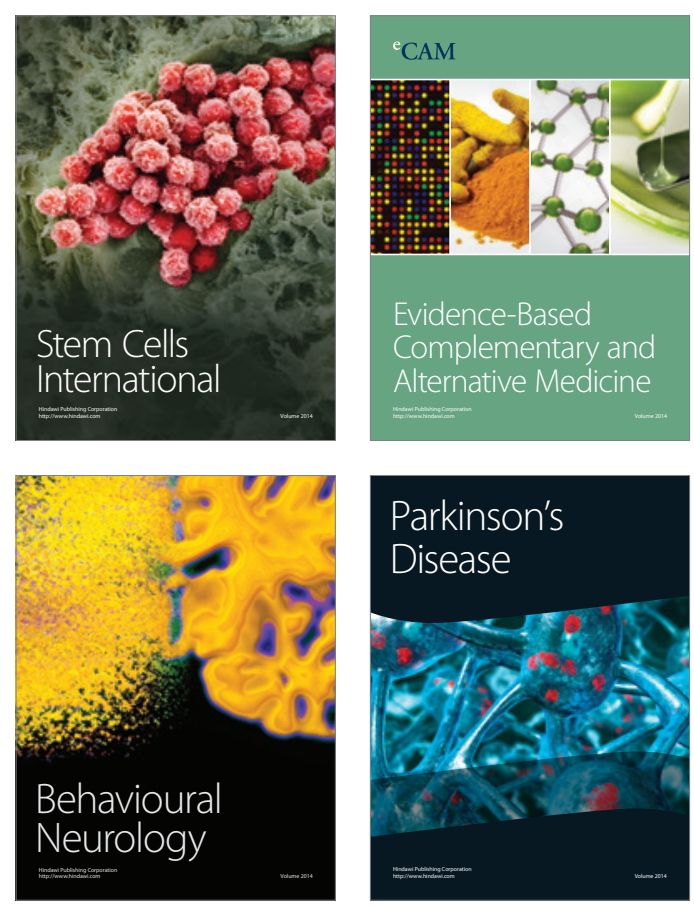

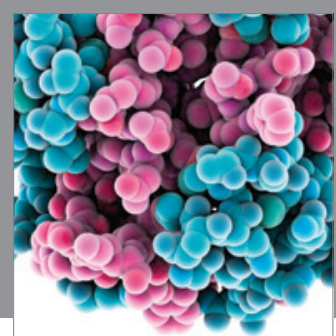

Journal of
Diabetes Research

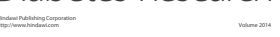

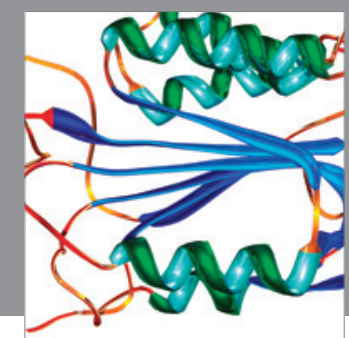

Disease Markers
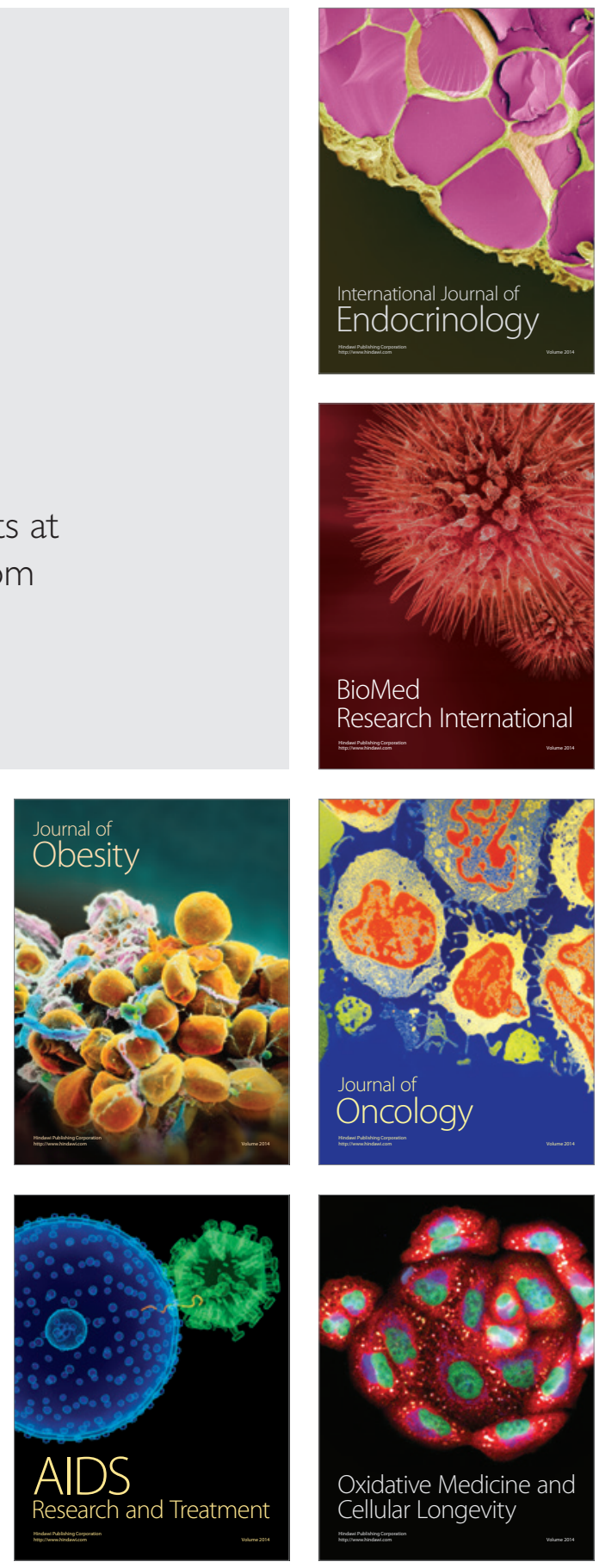